\title{
Global Stability Analysis of the Disease-Free Equilibrium State of a Mathematical Model of Trypanosomiasis
}

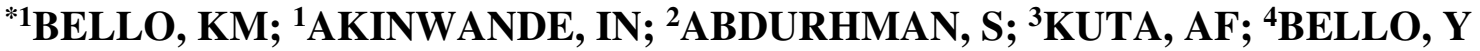 \\ ${ }^{l}$ Department of Mathematics, Federal University of Technology, Mina, Nigeria. \\ ${ }^{2}$ Department of Mathematics, Federal University Birnin Kebbi, Nigeria. \\ ${ }^{3}$ Department of Microbiology, Federal University of Technology Minna, Nigeria \\ ${ }^{4}$ Department of Mathematics, Federal Polytechnic, Bida .Nigeria \\ *Corresponding Author Email: qudratmusa62@gmail.com; Tel: +234 (0) 8163376642; +234 (0) 8059456596
}

\begin{abstract}
The global stability analysis represents a compound failure, mechanism which provides lower calculated factors of safety. In this research, the global stability analysis was used to propose a mathematically model of the transmission dynamics and control of Trypanosomiasis, known as African sleeping sickness. We obtained the Disease-free equilibrium state and present graphical profile of some of the compartments.
\end{abstract}

\section{DOI: https://dx.doi.org/10.4314/jasem.v23i2.1}

Copyright: Copyright (C) 2019 Bello et al. This is an open access article distributed under the Creative Commons Attribution License (CCL), which permits unrestricted use, distribution, and reproduction in any medium, provided the original work is properly cited.

Dates: Received: 31 October 2018; Revised: 05 January 2019; Accepted 03 February 2019

Keywords: Equilibrium state, trypanosome, sleeping sickness, stability

African Trypanosomiasis (AT) commonly called sleeping sickness is an infectious disease of both human beings and animals. It is a vector-borne parasitic disease caused by an extracellular protozoa belonging to the genus, trypanosome, species brucei. The parasites are transmitted to humans by tsetse fly (Glossina genus) bite which have acquired their infection from human beings or animals harboring the pathogenic parasite. World Health Organization $(2013$, 2015) reported that approximately 60 million people and 48 million cattle out of the estimated 172 million cattle are at risk of sleeping sickness in 36 countries in sub-Saharan Africa, In 1998 almost 40,000 cases were reported, but estimates were that between 300,000 to 500,000 were undiagnosed and therefore not treated.17, 600 cases were reported in 2004 , below 10,000 cases in 2009 , and it dropped to 6,314 cases in 2012.The disease ranked $3^{\text {rd }}$ in economic importance (after malaria and schistosomiasis) of all vector-borne human diseases and it is also the second greatest cause of mortality in affected communities, countries such as Angola, the Democratic Republic of Congo and Sudan had 50 percent occurrence in several of their villages. A number of mathematical models both simulation and analytical have been proposed to describe infectious diseases and African Trypanosomiasis. Otieno et al., (2014) describes an analytical model for trypanosomiasis in a cattle population. Damian et al.,
(2014) model the use of insecticide-treated cattle to control tsetse and trypanosome bruceirhodesiense, Madsen et al., (2012) described the effect of seasonal fluctuation in tsetse fly population and human African Trypanosomiasis, John et al.,(2012), model the Control of Trypanosomiasis using Trypanocides or Insecticide-Treated Livestock, Nannyonga et al., (2010) model on co-infection of malaria and trypanosomiasis. Akinwande (1995), Diekman (1990, 2000), Abdulrahman (2014) formulated models on infectious diseases. In this study, we describe a model for trypanosomiasis in human and cattle populations, which incorporates stage progression, screening and treatment of the population.

\section{MODEL FORMULATION}

A mathematical model of the dynamics of Trypanosomiasis incorporating stage progression, screening and treatment of the population, as control strategies was formulated. The model involved three interacting populations, humans, vectors and livestock. The total populations are compartmentalized intonine epidemiological classes, with the following variables: $S_{h}(t)=$ Susceptible humans at time $\mathrm{t} ; \mathrm{I}_{h}(\mathrm{t})=$ Infected humans first stage at time $\mathrm{t} ; \mathrm{I}_{m}(\mathrm{t})=$ Infected humans second stage at time $\mathrm{t} ; \mathrm{R}_{h}(\mathrm{t})=$ Recovered humans at time $\mathrm{t} ; \mathrm{N} \forall(\mathrm{t})=$ Non-carrier vectors at time $\mathrm{t} ; \mathrm{C}_{v}(\mathrm{t})=$ Carrier vectors at time $\mathrm{t} ; \mathrm{S}_{l}(\mathrm{t})=$ Susceptible livestock at time $\mathrm{t} ; \mathrm{I}_{l}(\mathrm{t})$ 
$=$ Infected livestock at time $\mathrm{t}$, and $\mathrm{R}_{l}(\mathrm{t})=$ Recovered livestock at time t.

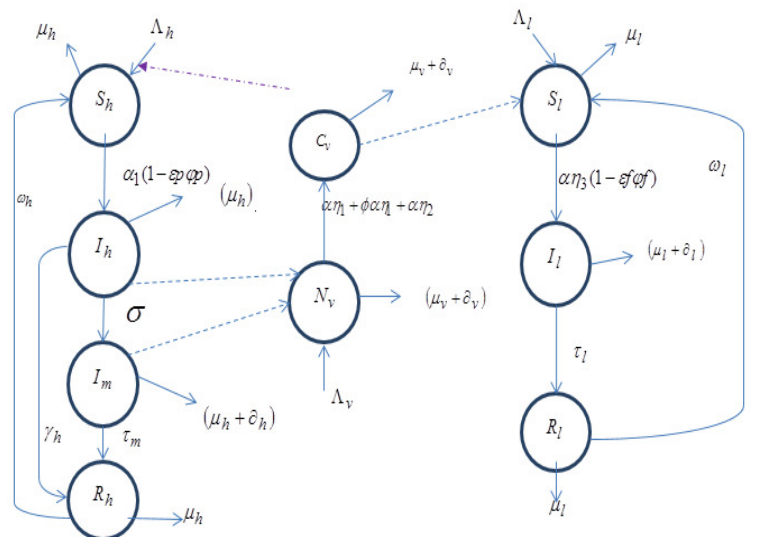

Fig 1: Schematic diagram of AT transmission dynamics and control.

The mathematical equations of the model can be described by a system of ordinary differential equations given below;

$$
\begin{aligned}
& \frac{d S_{h}}{d t}=\Lambda_{h}+\omega_{h} R_{h}-\frac{\alpha C_{v} S_{h}(1-\varepsilon p \varphi p)}{P_{h}}-\mu_{h} S_{h} \quad 1 \\
& \frac{d I_{h}}{d t}=\frac{\alpha C_{v} S_{h}(1-\varepsilon p \varphi p)}{P_{h}}-\left(\sigma+\gamma_{h}+\mu_{h}\right) I_{h} \\
& \frac{d I_{m}}{d t}=\sigma I_{h}-\left(\tau_{m}+\mu_{h}+\delta_{h}\right) I_{m} \\
& \frac{d R_{h}}{d t}=\gamma_{h} I_{h}+\tau_{m} I_{m}-\left(\mu_{h}+\omega_{h}\right) R_{h} \\
& \frac{d N_{v}}{d t}-\Lambda_{v}-\left(\frac{\alpha \eta_{1} I_{h}}{P_{v}}+\frac{\phi \alpha r \eta I_{m}}{P_{v}}+\frac{\alpha r_{12} I_{l}}{P_{v}}\right) N_{v}-\left(\mu_{v}+\delta_{v}\right) C_{v} \quad 5 \\
& \frac{d C_{v}}{d t}-\left(\frac{\alpha \eta_{1} T_{h}}{I_{v}^{\prime}}+\frac{\not \alpha \alpha \eta_{1} T_{m}}{!_{v}^{\prime}}+\frac{\alpha \eta_{2} T_{l}}{J_{v}^{\prime}}\right) N_{v}-\left(\mu_{v}+\mathcal{E}_{v}\right) C_{v} \quad 6 \\
& \frac{d I_{l}}{d t}=\frac{\alpha \eta_{3} C_{2} S_{l}(1-\varepsilon f \varphi f)}{P_{l}}-\left(\tau_{l} I_{l}+\mu_{l}+\delta_{l}\right) I_{l} \\
& \frac{d R_{l}}{d t}=\tau_{l} I_{l}-\left(\omega_{l}+\mu_{l}\right) R_{l} \\
& 8
\end{aligned}
$$

Where

$$
\left.\begin{array}{l}
P_{h}(t)=S_{h}(t)+I_{h}(t)+I_{m}(t)+R_{h}(t) \\
P_{l}(t)=S_{l}(t)+I_{l}(t)+R_{l}(t) \\
P_{v}(t)=N_{v}(t)+C_{v}(t)
\end{array}\right\}
$$

$\frac{d S_{l}}{d t}=\Lambda_{l}+\omega_{l} R_{l}-\frac{\alpha \eta_{3} C_{v} S_{l}(1-\varepsilon f \varphi f)}{P_{l}}-\mu_{l} s_{l}$

So that

$$
\left.\begin{array}{l}
\frac{d P_{h}}{d t}=\Lambda_{h}-\mu_{h} P_{h}-\delta_{h} I_{h}-\delta_{h} I_{m} \\
\frac{\mathrm{dP}_{1}}{\mathrm{dt}}=\Lambda_{l}-\mu_{l} P_{l}-\delta_{l} I_{l} \\
\frac{\mathrm{dP}_{\mathrm{v}}}{\mathrm{dt}}=\Lambda_{v}-\left(\mu_{v}+\delta_{v}\right) C_{v}
\end{array}\right\}
$$

In a biological region-feasible region $\Omega=\left\{S_{h} I_{h}, I_{m}, R_{h}, N_{v}, C_{v}, S_{l}, I_{l}, R_{l}\right\} \in \Re_{4}^{9}: N \leq P_{h}+P_{l}+P_{v} \quad 12$

Where the parameters

$\Lambda_{h}, \Lambda_{v}$ and $\Lambda_{l}$ are the daily recruitment rates of human, vector and livestock respectively into the susceptible population. $\alpha, \alpha \eta_{1}, \alpha \eta_{2}$ and $\alpha \eta_{3}$ are the effective transmission rates of AT from vector to human, human to vector, vector to livestock and livestock to vector while $\mu_{h}, \mu_{l}$ and $\mu_{v}, \delta_{h}, \delta_{l}$ and $\delta_{v}$ are the natural and induced death rates for human, livestock and vector populations. $\gamma_{h}$ is recovery rate of human due to natural healing, $\tau_{m}$ and $\tau_{l}$ are treatment rates of infected human and infected livestock respectively. $\omega_{h}, \omega_{l}$-waning rate of temporal immunity for human and livestock, $\varepsilon p$ is the efficacy of protective clothing, $\boldsymbol{\varepsilon}_{f}$ efficacy of fumigation, $\varphi_{p}$ human compliance with protective clothing and $\varphi_{f}$ rate of usage of fumigation. .

Disease-free Equilibrium $E^{0}$ : At disease -free equilibrium state there is no disease. Hence, the infected classes are zero. From (1-9)

$$
\begin{aligned}
& \Lambda_{h}+\omega_{h} R_{h} \frac{\alpha C_{h}^{*} S_{h}^{*}(1-\varepsilon p \varphi p)}{P_{h}^{*}}-\mu_{h} S_{h}^{*}=0 \\
& \frac{\alpha C_{h}^{*} S_{h}^{*}(1-\varepsilon p \varphi p)}{P_{h}^{*}}-K_{1} I_{h}^{*}=014 \\
& \sigma I_{h}^{*}-K_{2} I_{m}^{*}=0 \\
& \gamma_{h} I_{h}^{*}+\tau_{m} I_{m}^{*}-K_{3} R_{h}^{*}=0
\end{aligned}
$$




$$
\left.\frac{\alpha \eta_{1} I_{h}^{*} N_{v}^{*}}{P_{v}^{*}}+\frac{\phi \alpha \eta_{1} I_{m}^{*} N_{v}^{*}}{P_{v}^{*}}+\frac{\alpha \eta_{2} I_{l}^{*} N_{v}^{*}}{P_{v}^{*}}-K_{4} C_{v}^{*}=0 \quad 17 \quad \begin{array}{l}
K_{1}=\left(\sigma+\tau_{h}+\mu_{h}\right), K_{2}=\left(\tau_{m}+\mu_{h}+\delta_{h}\right), K_{3}=\left(\mu_{h}+\omega_{h}\right) \\
K_{4}=\left(\mu_{v}+\delta_{v}\right), K_{5}=\left(\tau_{l}+k_{l}+\delta_{l}\right), K_{6}=\left(k_{l}+\omega_{l}\right)
\end{array}\right\}
$$

$\Lambda_{v}-\left(\frac{\alpha \eta_{1} I_{h}^{*} N_{v}^{*}}{P_{v}^{*}}+\frac{\phi \alpha \eta_{1} I_{m}^{*} N_{v}^{*}}{P_{v}^{*}}+\frac{\alpha \eta_{2} I_{l}^{*} N_{v}^{*}}{P_{v}^{*}}\right)-K_{4} N_{v}^{*}=0$

$\Lambda_{l}+\omega_{l} R_{l}^{*}-\frac{\alpha \eta_{3} C_{v}^{*} S_{l}^{*}(1-\varepsilon f \varphi f)}{P_{l}^{*}}-k_{l} S_{l}^{*}=0$

From (15),we have

$$
I_{m}^{*}=\frac{\sigma I_{h}^{*}}{K_{2}}
$$

$\frac{\alpha \eta_{3} C_{v}^{*} S_{l}^{*}(1-\varepsilon f \varphi f)}{P_{l}^{*}}-K_{5} I_{l}^{*}=0 \quad 20$

$\tau_{l} I_{l}^{*}-K_{6} R_{l}^{*}=0$

Where
Substituting (23) into (16) yields

$$
R_{h}^{*}=\left(\frac{\gamma_{h} K_{2}+\tau_{m} \sigma}{K_{2} K_{3}}\right) I_{h}^{*}
$$

Substituting (24) into (13) gives

$$
S_{h}^{*}=\left[\frac{\Lambda_{h} K_{2} K_{3}+\omega_{h}\left(K_{2} \gamma_{h}+\tau_{m} \sigma\right) I_{h}^{*}}{K_{2} K_{3}\left\{\alpha C_{v}^{*}(1-\varepsilon p \varphi p)+\mu_{h} P_{h}^{*}\right\}}\right] P_{h}^{*}
$$

From (16), (23)and (24) we have

$$
I_{h}^{*}=\frac{\alpha C_{v}^{*}(1-\varepsilon p \varphi p) K_{2} K_{3} \Lambda_{h}}{\left\{\alpha\left[K_{1} K_{2} K_{3}-\omega_{h}\left(K_{2} \gamma_{h}+\tau_{m} \sigma\right)\right] C_{v}^{*}(1-\varepsilon p \varphi p)+K_{1} K_{2} K_{3} \mu_{h} P_{h}^{*}\right\}} \quad 26
$$

Let

$$
\begin{aligned}
& \begin{array}{l}
A=\alpha\left[K_{1} K_{2} K_{3}-\omega_{h}\left(K_{2} \gamma_{h}+\tau_{m} \sigma\right)\right](1-\varepsilon p \varphi p) \\
B=K_{1} K_{2} K_{3} \mu_{h} P_{h}^{*}
\end{array} \\
& I_{h}^{*}=\frac{K_{2} K_{3} \alpha \Lambda_{h} C_{v}^{*}}{A C_{v}^{*}+B}
\end{aligned}
$$

Put (27) into (24) gives

$$
R_{h}^{*}=\left(\gamma_{h} K_{2}+\tau_{m} \sigma\right)\left(\frac{\alpha \Lambda_{h} C_{v}^{*}}{A C_{v}^{*}+B}\right) \quad 28
$$

Also by putting (19) into (17) gives

$$
S_{h}^{*}=\left[\frac{K_{2} K_{3}\left[\Lambda_{h}\left(A C_{v}^{*}+B\right)+\omega_{h}\left(K_{2} \gamma_{h}+\tau_{m} \sigma\right) \alpha \Lambda_{h} C_{v}^{*}\right.}{K_{2} K_{3}\left(\alpha C_{v}^{*}+\mu_{h} P_{h}^{*}\right)\left(A C_{v}^{*}+B\right)}\right] P_{h}^{*} \quad 29
$$

From (20)

$$
I_{l}^{*}=\frac{\alpha \eta_{3} C_{v}^{*} s_{l}^{*}(1-\varepsilon f \varphi f)}{K_{5} P_{l}^{*}}
$$

Put (30) into (21) gives

$$
R_{l}^{*}=\frac{\tau_{l} \alpha \eta_{3} S_{l}^{*}(1-\varepsilon f \varphi f) C_{v}^{*}}{K_{5} K_{6} P_{l}^{*}}
$$

From (30), (19) becomes 


$$
S_{l}^{*}=\frac{K_{5} K_{6} P_{l}^{*} \Lambda_{l}}{\alpha \eta_{3} C_{v}^{*}(1-\varepsilon f \varphi f) K_{5} K_{6}-\omega_{l} \tau_{l} \alpha \eta_{3} C_{v}^{*}(1-\varepsilon f \varphi f)+K_{5} K_{6} P_{l}^{*} k_{l}} \quad 32
$$

Similarly substituting (27) into (23) gives

$$
I_{m}^{*}=\frac{\sigma \alpha \Lambda_{h} K_{3} C_{v}^{*}}{A C_{v}^{*}+B} \quad 33
$$

Also (32) into (31) gives

$$
R_{l}^{*}=\frac{\tau_{l} \alpha \eta_{3} C_{v}^{*}(1-\varepsilon f \varphi f)}{K_{5} K_{6} P_{l}^{*}}\left[\frac{K_{5} K_{6} P_{l}^{*} \Lambda_{l}}{K_{5} K_{6} \alpha \eta_{3} C_{v}^{*}(1-\varepsilon f \varphi f)-\omega_{l} \tau, \alpha \eta_{3} C_{v}^{*}(1-\varepsilon f \varphi f)+K_{5} K_{6} P_{l}^{*} k_{l}}\right]
$$

Also (32) into (30) yields

$$
I_{l}^{*}=\frac{\alpha \eta_{3} C_{v}^{*}(1-\varepsilon f \varphi f)}{K_{5} P_{l}^{*}}\left[\frac{K_{5} K_{6} P_{l}^{*} \Lambda_{l}}{K_{5} K_{6} \alpha \eta_{3} C_{v}^{*}(1-\varepsilon f \varphi f)-\omega_{l} \tau, \alpha \eta_{3} C_{v}^{*}(1-\varepsilon f \varphi f)+K_{5} K_{6} P_{l}^{*} k_{l}}\right]
$$

Substituting and simplifying (17)

$$
N_{v}=\frac{\Lambda_{v} P_{v}^{*}\left(C_{v}^{*}+B\right)}{\alpha^{2} \eta_{1} \Lambda_{h} K_{3}\left(K_{2}+\phi \sigma\right) C_{v}^{*}}+\frac{\Lambda_{v} P_{v}^{*}}{K_{4} P_{v}^{*}}+\frac{\Lambda_{v} P_{v}^{*}\left\{K_{5} P^{*}\left[\alpha \eta_{3}\left(K_{5} K_{6}-\omega_{l} \tau_{l}\right) C_{v}^{*}(1-\varepsilon f \varphi f)+K_{5} K_{6} P^{*} k_{l}\right\}\right.}{\alpha^{2} \eta_{2} \eta_{3} C_{v}^{*}(1-\varepsilon f \varphi f) K_{5} K_{6} P_{l}^{*} \Lambda_{l}}
$$

From (18), we have

$$
C_{v}^{*}\left\{\frac{\alpha^{2} \eta_{1} \Lambda_{h} K_{3}\left(K_{2}+\phi \sigma\right) N_{v}^{*}}{\left(A C_{v}^{*}+B\right) P_{v}^{*}}+\frac{\alpha^{2} \eta_{3} \eta_{4} K_{5} K_{6} P_{l}^{*} \Lambda_{l} N_{v}^{*}}{P_{v}^{*} K_{5} P_{l}^{*}\left[\alpha \eta_{3}\left[\left(K_{5} K_{6}-\omega_{l} \tau_{l}\right) C_{v}^{*}\right]+K_{5} K_{6} P_{l}^{*} \mu_{l}\right.}-K_{4}\right\}=0
$$

Thus

$$
\begin{aligned}
& C_{v}^{*}=0 \text { or } \\
& \frac{\alpha^{2} \eta_{1} \Lambda_{h} K_{3}\left(K_{2}+\phi \sigma\right) N_{v}^{*}}{\left(A C_{v}^{*}+B\right) P_{*}}+\frac{37}{P_{v}^{*} K_{5} P_{l}^{*}\left[\alpha \eta_{3}\left[\left(K_{5} K_{6}-\omega_{l} \tau_{l}\right) C_{v}^{*}\right]+K_{5} K_{6} P_{l}^{*} \mu_{l}\right.}-K_{4}=0 \quad 38
\end{aligned}
$$

Substituting (37) into (27),(28), (33),(34),(35) we obtain

$$
I_{h}^{*}=I_{m}^{*}=I_{l}^{*}=R_{h}^{*}=R_{l}^{*}=0
$$

$C_{v}^{*}>0$

when

$$
\frac{\alpha^{2} \eta_{1} \Lambda_{h} K_{3}\left(K_{2}+\phi \sigma\right) N_{v}^{*}}{K_{4}\left(A C_{v}^{*}+B\right) P_{v}}+\frac{\alpha^{2} \eta_{2} \eta_{3} K_{5} K_{6} P_{l} \Lambda_{l} N_{v}^{*}}{K_{4} P_{v} K_{5} P_{l}\left[\alpha \eta_{3}\left[\left(K_{5} K_{6}-\omega_{l} \tau_{l}\right) C_{v}^{*}\right]+K_{5} K_{6} P_{l} \mu_{l}\right.}>1
$$

Thus giving two different equilibrium state, DFE state where $I_{h}^{*}=I_{m}^{*}=I_{l}^{*}=C_{v}^{*}=0$ 
And endemic equilibrium where all the compartments are greater than zero

Now consider (37), substituting into (13),(17), and (19),we have

$$
S_{h}^{*}=\frac{\Lambda_{h}}{\mu_{h}}, \quad N_{v}^{*}=\frac{\Lambda_{v}}{K_{4}} \quad S_{l}^{*}=\frac{\Lambda_{l}}{\mu_{l}}
$$

Thus a DFE state of the model exists at the point

$$
\left(\left(S_{h}^{*}, I_{h}^{*}, I_{m}^{*}, R_{h}^{*}, N_{v}^{*}, C_{v}^{*}, S_{l}^{*}, I_{l}^{*}, R_{l}^{*}\right)=\left(\frac{\Lambda_{h}}{\mu_{h}}, 0,0,0, \frac{\Lambda_{v}}{K_{4}}, 0, \frac{\Lambda_{l}}{\mu l}, 0,0\right)\right.
$$

stable if $R_{c}<1$

Effective Reproduction number, $R_{c}$ : We apply the next generation matrix operator as used by Diekmann and Heesterbeek (2000), and improved upon by Van de Driessche and Watmough (2002), we obtained the effective reproduction number $\mathrm{R}_{\mathrm{c}}=\mathrm{FV}^{-1}$ where $\mathrm{F}$ is the matrix of new infection terms and $\mathrm{V}$ is the matrix of the transmission terms formed from the coefficient of the infected classes $\left(\mathrm{I}_{\mathrm{m}}, \mathrm{I}_{\mathrm{h}}, \mathrm{I}_{1}, \mathrm{C}_{\mathrm{v}}\right)$.

$$
\begin{aligned}
& F=\left[\begin{array}{cccc}
0 & 0 & \alpha(1-\varepsilon p \varphi p) & 0 \\
0 & 0 & 0 & 0 \\
\alpha \eta_{1} & \phi \alpha \eta_{1} & 0 & \alpha \eta_{2} \\
0 & 0 & \alpha \eta_{3}(1-\varepsilon f \varphi f) & 0
\end{array}\right] \text { and }_{V^{-1}}=\left[\begin{array}{cccc}
\frac{1}{k_{1}} & 0 & 0 & 0 \\
\frac{\alpha}{K_{1} K_{2}} & \frac{1}{K_{2}} & 0 & 0 \\
0 & 0 & \frac{1}{K_{4}} & 0 \\
0 & 0 & 0 & \frac{1}{K_{5}}
\end{array}\right] 41 \\
& F V^{-1}=\left[\begin{array}{cccc}
0-\lambda & 0 & \frac{\alpha(1-\varepsilon p \varphi p)}{K_{4}} & 0 \\
0 & 0-\lambda & 0 & 0 \\
\frac{\alpha \eta_{1}}{K_{1}}+\frac{\phi \sigma \alpha \eta_{1}}{K_{1} K_{2}} & \frac{\phi \alpha \eta_{1}}{K_{2}} & 0-\lambda & \frac{\alpha \eta_{2}}{K_{5}} \\
0 & 0 & \frac{\alpha \eta_{3}(1-\varepsilon f \varphi f)}{K_{4}} & 0-\lambda
\end{array}\right] 42
\end{aligned}
$$

From which we obtained the effective reproduction number as

$R_{c}=\sqrt{\frac{\alpha^{2} \eta_{1} K_{5}(1-\varepsilon p \varphi p)\left[K_{2}+\phi \sigma\right]+K_{1} K_{2} \alpha^{2} \eta_{2} \eta_{3}(1-\varepsilon f \varphi f)}{K_{1} K_{2} K_{4} K_{5}}}$

\section{Global Stability of Disease-Free equilibrium, $\mathbf{E}^{\mathbf{0}}$ :}

Theorem 1: The DFE $E^{O}$ of model equation (1) is globally asymptotically stable (GAS) in $\Omega$ if $R_{c}<1$ and unstable if $R_{c}>1$.

Proof: - To establish the global stability of the DFE, the two conditions for the global stability of DFE as in Castillo-Chavel et al (2002), for $\mathrm{R}_{\mathrm{c}}<1$ was used for the equations of model system (1-9). It is possible to re write the model system (1-9) in the following way

$$
\left.\begin{array}{rl}
\frac{d X_{s}}{d t} & =A\left(X_{S}-X_{D F E, S}\right)+A_{1} X_{i} \\
\frac{d X_{i}}{d t} & =A_{2} X_{i}
\end{array}\right\} 43
$$


Where $X_{s}=\left(S_{h}^{0}, R_{h}^{0}, N_{v}^{0}, S_{l}^{0}, R_{l}^{0}\right)^{T}$ denote the different compartments of non - infected individuals, and $X_{i}=\left(I_{h}^{0}, I_{m}^{0}, C_{v}^{0}, I_{l}^{0}\right)^{T}$ denotes the compartments of different infected individuals. The disease - free equilibrium is denoted as $E^{O}=\left(X_{S}^{*}, O\right)$. where $X_{S}^{*}=\left(P_{h}^{O}+P_{l}^{O}+P_{v}^{O}, 0\right)$

$\frac{d X_{s}}{d t}=F\left(X_{s}, 0\right)=\left\{\begin{array}{l}\Lambda_{h}+\omega_{h} R_{1}^{0}-\mu_{h} S_{h}^{0} \\ -\left(\mu_{h}+\omega_{h}\right) R_{h}^{0} i . e .-K_{3} R_{h}^{0} \\ \Lambda_{v}-K_{4} N_{v}^{0} \\ \Lambda_{l}+\omega_{l} R_{l}^{0}-k_{l} S_{l}^{0} \\ -K_{6} R_{l}^{0}\end{array}\right.$

a linear differential equation which on solving gives the following:

$$
\begin{array}{ll}
S_{h}^{0}(t)=\frac{\left(\Lambda_{h}+\omega_{h} R_{h}^{0}\right)}{\mu_{h}}-\frac{\left(\Lambda_{h}+\omega_{h} R_{h}^{0}\right) e^{-\mu_{h} t}}{\mu_{h}}+S_{h}^{0}(0) e^{-\mu_{h} t} & 45 \\
R_{h}^{0}(t) & =R_{h}^{0}(0) e^{-k_{3} t} \\
N_{v}^{0}(t) & =\frac{\Lambda_{v}}{4}-\frac{\Lambda_{v} e^{-k_{4} t}}{4}+N_{v}^{0}(0) e^{-k_{4} t} \\
S_{l}^{0}(t) & =\frac{\left(\Lambda_{l}+\omega_{l} R_{l}^{0}\right)}{k_{l}}-\frac{\left(\Lambda_{l}+\omega_{l} R_{l}^{0}\right) e^{-k_{l} t}}{k_{l}}+S_{l}^{0}(0) e^{-k_{l} t} \\
R_{l}^{0}(t) & =R_{l}^{0}(0) e^{-K_{6} t}
\end{array}
$$

Now from (10) gives

$S_{h}^{0}(t)+R_{h}^{0}(t)+N_{v}^{0}(t)+S_{l}^{0}(t)+R_{l}^{0}(t) \rightarrow N^{0}(t), \quad$ as $\quad \mathrm{t} \rightarrow 0$.regardless of the value of $S_{h}^{o}(o), R_{h}^{o}(o), N_{v}^{o}(o), S_{l}^{o}(o), R_{l}^{o}(o)$.Thus $X_{S}^{*}=\left(P_{h}^{o}+P_{l}^{o}+P_{v}^{o}, 0\right)$ is globally and asymptotically stable.

Next, $\overline{\mathrm{G}}\left(X_{s}, X_{i}\right)=A X_{i}-\mathrm{G}\left(X_{s}, X_{i}\right)$,

$$
A X i=\left[\begin{array}{cccc}
-K_{1} & 0 & \alpha(1-\varepsilon p \varphi p) & 0 \\
\sigma & -K_{2} & 0 & 0 \\
\alpha \eta_{1} & \phi \alpha \eta_{1} & -K_{4} & \alpha \eta_{2} \\
0 & 0 & \alpha \eta_{3}(1-\varepsilon f \varphi f) & -K_{5}
\end{array}\right]
$$

It is obvious that this is an M-matrix (Metzler also called quasi-positive matrix whose diagonal Elementsare non-negative). The off diagonal elements of A are non-negative.

$$
G\left(X_{s}, X_{i}\right)=\left[\begin{array}{cc}
\frac{\alpha C_{v}^{0} S_{h}^{0}(1-q p \varphi p)}{P_{h}} & -K_{1} I_{h}^{0} \\
\sigma I_{h}^{0} & -K_{2} I_{m}^{0} \\
\frac{\alpha \eta_{1} I_{h}^{0}}{P_{v}}+\frac{\phi \alpha \eta_{1} I_{m}^{0}}{P_{v}}+\frac{\alpha \eta_{2} I_{l}^{0}}{P_{v}} & -K_{4} C_{v}^{0} \\
\frac{\alpha \eta_{3} C_{v}^{0} S_{l}^{0}(1-q f \varphi f)}{P_{l}} & -K_{5} I_{l}^{0}
\end{array}\right]
$$


Then,

$\hat{G}\left(X_{s}, X_{i}\right)=A X_{s}-G\left(X_{s}, X_{i}\right)=\left[\begin{array}{l}0 \\ 0 \\ 0 \\ 0\end{array}\right]^{T} 52$

i.e.

$$
\hat{G}\left(X_{s} X_{i}\right)=\left[\begin{array}{llll}
0 & 0 & 0 & 0
\end{array}\right]^{T}
$$

Thus, $\hat{G}\left(X_{s}, X_{i}\right)=0$,hence the proof is complete.

Numerical Simulations: We give the numerical simulation for the model system (1-9) for the purpose of verifying some of the analytic results. We give the numerical simulation for the model system (1) for the purpose of verifying some of the analytic results.

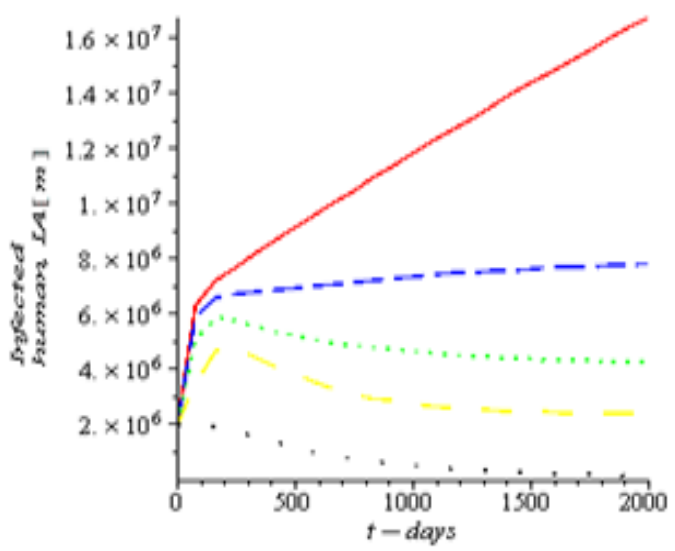

Fig 2: Graph of infected human IA(t) against time $\mathrm{t}$ when $\varphi p=\varphi f=\delta_{v}=\tau_{m}=\tau_{l}=0, \varphi p=\varphi f=\delta_{v}=\tau_{m}=\tau_{l}=0.25$, $\varphi p=\varphi f=\delta_{v}=\tau_{m}=\tau_{l}=0.50, \varphi p=\varphi f=\delta_{v}=\tau_{m}=\tau_{l}=0.75$ $\varphi p=\varphi f=\delta_{v}=\tau_{m}=\tau_{l}=0.95$

Conclusion: In this paper, a non-linear mathematical model of AT is developed and analyzed incorporating the treatment of the infectious second stage human population, basic reproduction number $R_{O}$ was used to establish the conditions for Global Stability of the Disease-Free Equilibrium (DFE) showing that the Disease-Free Equilibrium will be logically asymptotically stable if $R_{0}<1$. Numerical simulation shows that the disease can be eradicated when both preventive and treatment strategies are adopted.

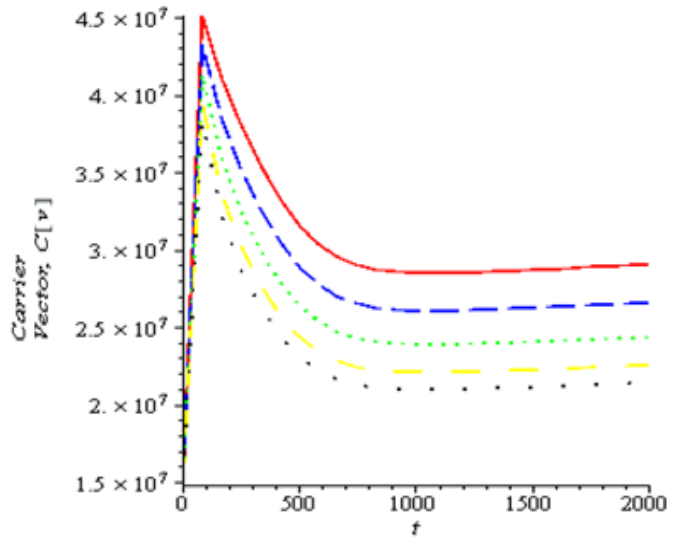

Fig 3: Graph of carrier vector at time $\mathrm{t}$ when $\delta_{v}=0, \delta_{v}=0.25, \delta_{v}=0.50, \delta_{v}=0.75, v=0.95$

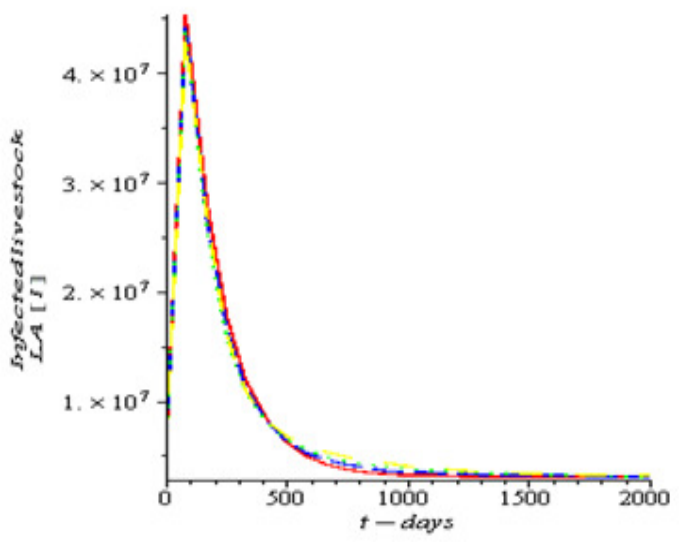

Fig.4: Graph of infected livestock I ( $\mathrm{t})$ against time when $\varphi p=\varphi f=\delta_{v}=\tau_{m}=\tau_{l}=0, \varphi p=\varphi f=\delta_{v}=\tau_{m}=\tau_{l}=0.25$ $\varphi p=\varphi f=\delta_{v}=\tau_{m}=\tau_{l}=0.50, \varphi p=\varphi f=\delta_{v}=\tau_{m}=\tau_{l}=0.75$

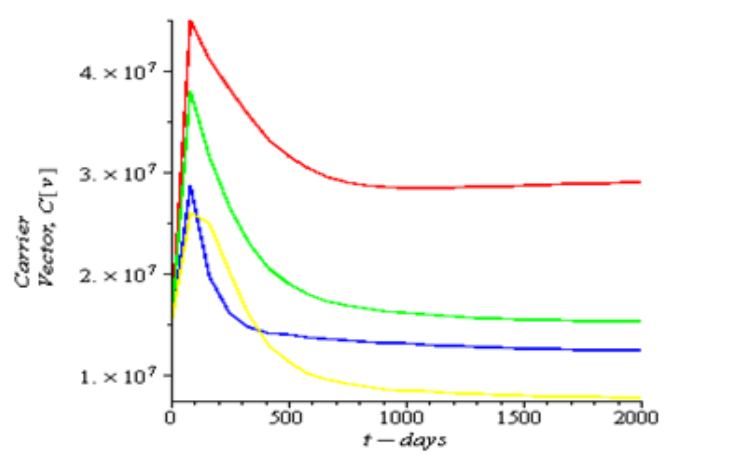

Fig 5: Graph of Carrier vector using combined strategies when 
$\varphi p=\varphi f=\delta_{v}=\tau_{m}=\tau_{l}=0, \varphi p=\varphi f=\delta_{v}=\tau_{m}$ Iotm $=02 \mathrm{~S}$ Rachid,O; Damian, K; Glyn, AV; $\varphi p=\varphi f=\delta_{v}=\tau_{m}=\tau_{l}=0.50, \varphi p=\varphi f=\delta_{v}=\tau_{m}=$ Stephen, JT (2012). The Modeling the Control of

\section{REFRENCES}

Abdurahman, S (2014). A Mathematical Model for the Transmission Dynamic and Control of Hepatitis B Virus. PhD Thesis Department of Mathematics \& Statistics. FUT Minna)

Akinwande, NI (1995). Local Stability Analysis of Equilibrium State of a Mathematical Model of Yellow Fever Epidemics. J. Nig. Math. Soc. Vol.6.

Diekmann, O; Heesterbeek, JP; Metz, AJ (1990). On the Definition and Computation of the Basic Reproduction number $\mathrm{R}_{0}$ in Model for infection Diseases in Heterogeneous Populations. J Math. Biol. 28: 365-382

Diekmann, O; Heesterbeek, JP (2000). Mathematical Epidemiology of Infectious Diseases Modeling Analysis and Interpretation. John Wiley and Sons Ltd. New York. U.S.A

Driessche, VP; Watmough, J (2002).Reproduction Number and sub-threshold Endemic Equilibria for Compartmental Models of Diseases Transmission. Math. Biosci. 180 (2002): 29-48. Insecticide-Treated Livestock. PLOS-Neglected Tropical Diseases (2012)

Nannyonga, B; Mugisha, JF; Luboobbi, LS (2010). Does Co-infection with Malaria BoostPersistence of Trypanosomiasis? Elsevier.Non-Linear Analysis: Real World Applications Journal homepage. Pp1-12 (col.fif.N.L) doi, $10.1016 \quad$ (J. Nonrma.2011.11.022. articles in- press.

Otieno, J; Mugisha, JT; Nannyongs, B; Oleche P (2014).Parameter Driven Dynamics of Trypanosomiasis in Cattle Population.Applied Math. Sciences, vol.8, No. 54: 2665-2685. Hikari Ltd.

World Health Organization (2013).Control and Surveillance of HAT. Report of WHO Expert Committee. WHO technical report series 984 Geneva Switzerland.

World Health Organization (2015).TrypanosomiasisHuman African (Sleeping Sickness).Fact Sheet $\mathrm{N}^{0} 259$. Update May 2015. 